\title{
Endogenous toxic metabolites and implications in cancer therapy
}

\author{
Namgyu Lee ${ }^{1} \cdot$ Meghan E. Spears $\mathbb{1}^{1} \cdot$ Anne E. Carlisle $\mathbb{I}^{1} \cdot$ Dohoon Kim $\mathbb{D}^{1}$
}

Received: 1 May 2020 / Revised: 16 June 2020 / Accepted: 15 July 2020 / Published online: 24 July 2020

(c) The Author(s) 2020. This article is published with open access

\begin{abstract}
It is well recognized that many metabolic enzymes play essential roles in cancer cells in producing building blocks such as nucleotides, which are required in greater amounts due to their increased proliferation. On the other hand, the significance of enzymes in preventing the accumulation of their substrates is less recognized. Here, we outline the evidence and underlying mechanisms for how many metabolites normally produced in cells are highly toxic, such as metabolites containing reactive groups (e.g., methylglyoxal, 4-hydroxynonenal, and glutaconyl-CoA), or metabolites that act as competitive analogs against other metabolites (e.g., deoxyuridine triphosphate and 1-2-hydroxyglutarate). Thus, if a metabolic pathway contains a toxic intermediate, then we may be able to induce accumulation and poison a cancer cell by targeting the downstream enzyme. Furthermore, this poisoning may be cancer cell selective if this pathway is overactive in a cancer cell relative to a nontransformed cell. We describe this concept as illustrated in selenocysteine metabolism and other pathways and discuss future directions in exploiting toxic metabolites to kill cancer cells.
\end{abstract}

\section{Introduction}

Metabolism is an aspect of cancer biology that is attractive in terms of therapy. First, it has been known for a long time that the metabolism of cancer cells differs from that of normal cells in many ways. A widely known metabolic alteration in cancer cells is high glucose consumption and high levels of lactate production with a lack of oxidative phosphorylation, referred to as the Warburg effect [1, 2]. Another commonly observed metabolic perturbation in cancer cells is the deregulated uptake of amino acids [3]. In particular, many cancer cells are highly dependent on glutamine for their survival and proliferation [3]. In addition, lipid metabolism is also modified in cancer cells [4] because rapidly proliferating cells require fatty acids for the synthesis of signaling molecules and membranes [5]. The identification of such cancer-specific metabolic changes provides the opportunity to develop novel therapeutic strategies to treat cancer. The "druggability" of enzymes further adds to the appeal of cancer metabolism as a therapeutic avenue. Even if cancer-selective targets are identified by

Dohoon Kim

Dohoon.Kim@umassmed.edu

1 Department of Molecular, Cell and Cancer Biology, University of Massachusetts Medical School, Worcester, MA 01605, USA characterizing the role of the targets in cancer, it can be difficult to translate the basic research into the clinic if the targets are not easily druggable, i.e., have small, hydrophobic pockets in regions required for their activity. Enzymes are, by their catalytic nature, highly druggable, due to their pockets for their substrates and coenzymes $[6,7]$.

Much of cancer metabolism research has centered on the idea of targeting the "cellular building blocks" that cancer cells require, and there are notable examples of clinical efficacy (Fig. 1a). Cancer cells upregulate a variety of metabolic pathways involved in the production of cellular building blocks that support the increased demand for the biosynthesis of proteins, lipids, and nucleic acids [8]. Antifolates, folate analogs that inhibit de novo nucleotide synthesis enzymes [9, 10], were among the very first chemotherapeutics. Since then, many additional therapies that inhibit nucleotide synthesis have been developed and are still used in the clinic to treat several cancers [11]. Two important examples are the use of 5-fluorouracil, which disrupts thymidine synthesis through the enzyme thymidylate synthase and gemcitabine, which can incorporate into DNA and targets deoxyribonucleotide synthesis through the enzyme ribonucleotide reductase, both of which are required for essential DNA synthesis in rapidly growing cancer cells [11]. In addition to targeting nucleotide synthesis, other biosynthetic pathways have been actively explored and have shown promise in preclinical models, 
A

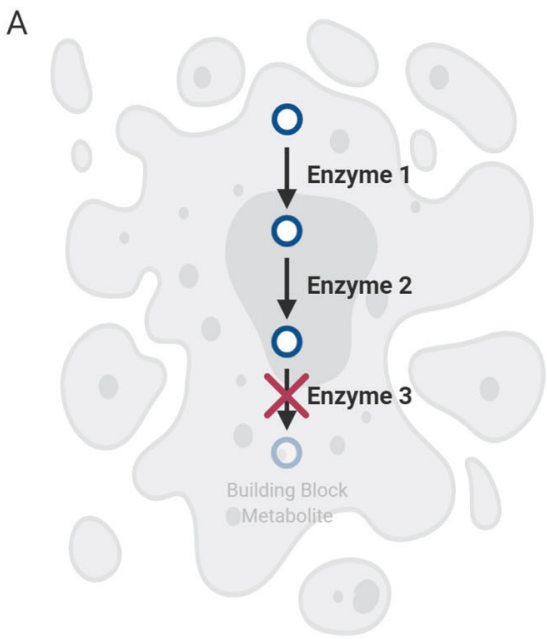

B

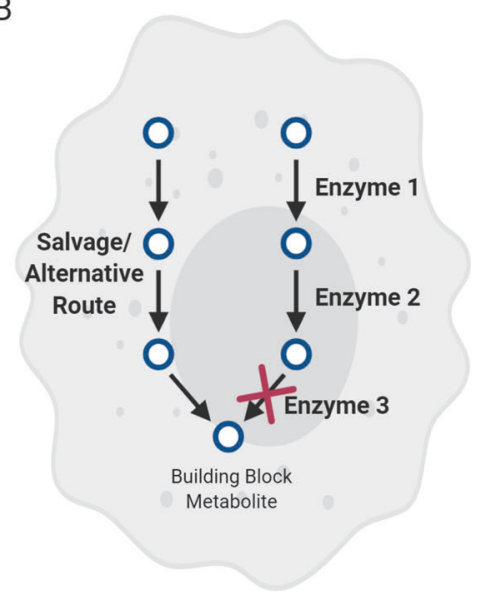

C

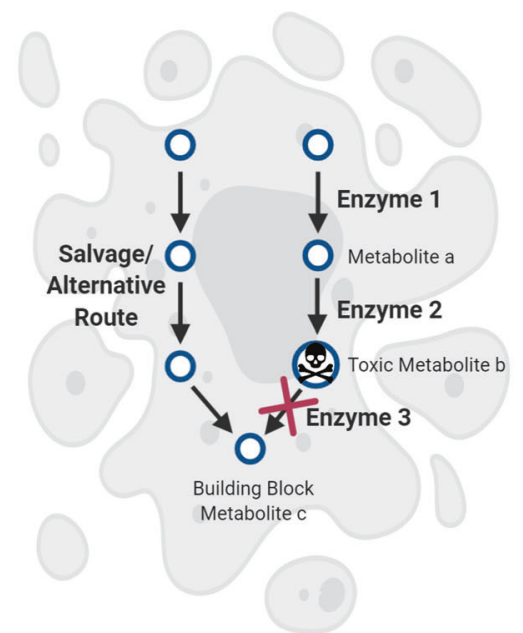

Fig. 1 Scenarios for targeting metabolic enzymes that produce essential cellular building blocks in cancer. a Targeting a metabolic enzyme to disrupt the production of a metabolite that is essential to a cancer cell can be an effective therapeutic strategy. b When there are alternate means for production or acquisition of an essential metabolite, targeting the synthesizing enzyme may be inadequate to kill a cancer cell. c An alternative approach is to target an enzyme directly downstream of a toxic metabolite, which will result in accumulation of the upstream toxic metabolite. Even if there are alternative routes for producing the building block metabolite, this strategy should still work to exert toxicity in a cancer cell. such as PHGDH required for serine biosynthesis and FASN required for fatty acid biosynthesis [12-14].

While the approach of starving a cancer cell of essential metabolites is both logical and proven, there are also some important factors that can limit the effectiveness of this strategy in killing a cancer cell. First, when the biosynthetic pathway for an essential metabolite is disrupted, there may be mechanisms by which a cell can salvage it through a secondary route (Fig. 1b). This can occur by compensatory production of the metabolite through an alternative pathway, uptake from the extracellular environment, or breakdown of existing macromolecules in the cell [8]. Such salvage pathways exist for many key metabolic pathways in the cell, such as nucleotide and sphingolipid biosynthesis. For both of these pathways, in addition to the de novo biosynthesis of essential intermediates like purines/pyrimidines and ceramides, respectively, cells can also obtain these metabolites through extracellular uptake from what is obtained in the diet, as well as degradation of more complex molecules in the cell such as nucleic acids and sphingomyelin [11, 15]. Cancer cells can also upregulate processes such as macropinocytosis [16] and autophagy [8], further contributing to their metabolic flexibility in obtaining building blocks. Therefore, the potential for multiple sources of essential building blocks can make targeting a biosynthetic enzyme insufficient to limit a metabolite adequately to kill a cancer cell.

Second, a metabolic enzyme that appears to be essential in tissue culture may not end up being essential in a more physiological (in vivo) context, as typical culture media conditions do not accurately reflect the diversity and quantity of metabolites that are available in the human bloodstream [17]. Uric acid, pyruvate, taurine and various other metabolites are present at higher levels in human serum than in culture media [17]. Consequently, disrupting a biosynthetic pathway may sufficiently deprive cells of an essential metabolite in tissue culture, while in vivo the cells may be able to obtain the metabolite from the bloodstream.

As illustrated, there are multiple reasons why targeting a particular enzyme that produces cellular building blocks may not be effective to kill a cancer cell. Thus, even when this enzyme appears to be important to a cancer cell, either due to the product it makes or due to it being increased in expression and/or activity, targeting it may not translate to effective therapy. Here, we outline an alternative approach: the notion of killing cancer cells not by impairing the production of biosynthetic building blocks, but rather by inducing an accumulation of toxic intermediates that lie within metabolic pathways. Assuming a standard linear metabolic pathway that involves the stepwise chemical conversion of a series of metabolites, the loss of an enzyme that converts toxic metabolite "b" to a nontoxic metabolite "c" may result in the accumulation of "b" to toxic levels in the cell (Fig. 1c). This approach may overcome some of the aforementioned limitations of the building block deprivation approach. Even if alternate synthesis or uptake routes exist for the building block synthesis, it cannot remedy the issue of accumulated toxic metabolites. Importantly, if this metabolic pathway is more active in a particular type of cancer cell compared with a normal cell, then the accumulation of "b" should occur at a greater rate in the cancer cell, providing a therapeutic window. The more toxic 

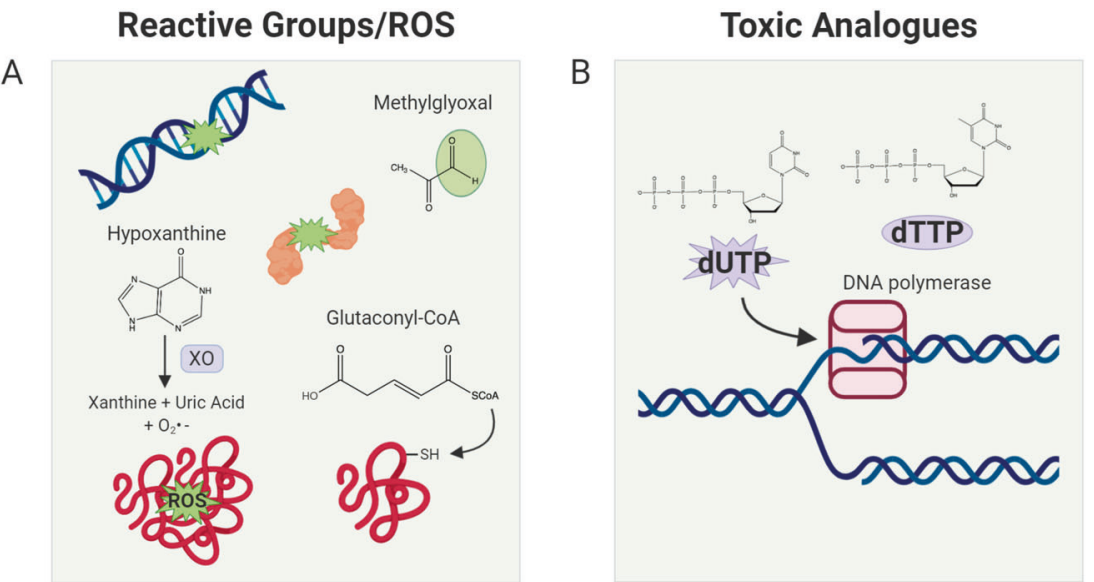

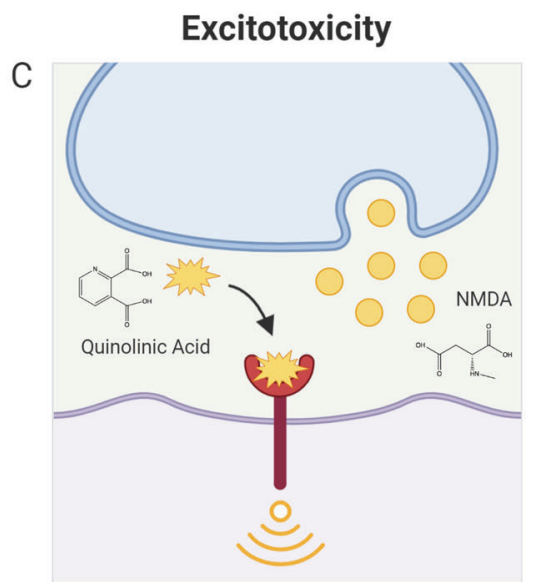

Fig. 2 Different categories of toxic metabolites based on their toxicity mechanism. a ROS/reactive groups. Some metabolites contain reactive groups such as aldehydes which allow them to form adducts with macromolecules such as DNA and proteins. Others can form reactive oxygen species and similarly damage DNA and proteins through oxidation. The representative ROS forming/reactive metabolites are depicted in the figure. Hypoxanthine is a ROS former, as it produces ROS as it is catabolized. Methylglyoxal is a reactive aldehyde. Glutaconyl-CoA is a reactive metabolite that preferentially reacts with thiol groups. b Toxic analogs. Due to the promiscuity of

metabolite "b" is, the more likely it is that the disruption of the downstream enzyme will be detrimental to the cancer cell.

First, we describe many metabolites normally produced in our cells that have highly toxic properties. We illustrate potential mechanisms for their toxicity, such as the presence of highly reactive groups that allow them to covalently modify cellular components, or their similarity to other metabolites that make them de facto competitive inhibitors. Second, we describe recent findings that demonstrate this approach, with an emphasis on the selenocysteine biosynthesis pathway, which we have recently shown to allow cancer cell-specific poisoning. Finally, we discuss future directions for applying this approach across many metabolic pathways to uncover novel Achille's heels in different cancers.

\section{Toxic metabolites}

It may seem counterintuitive that endogenously produced metabolites, which in many cases form the building blocks of the cell, can be poisonous. However, when cross referencing known metabolites with toxicity databases such as TOXNET [18] or HSDB [19], it becomes clear that many metabolites have toxic profiles. As a striking example, the commonly utilized fixative and hazard substance formaldehyde is produced naturally in the brain via oxidative breakdown of the folate backbone [20]. It can be inferred that under normal physiological conditions, the levels of metabolic enzymes and structural similarity of small metabolites, several metabolites confer their toxicity to cells via misincorporation of wrong nucleotides or competitive inhibition of the enzyme reactions. High dUTP/dTTP ratio promotes misincorporation of dUTP, which leads to DNA damage and eventually cell death cexcitotoxicity. Several metabolites can act as a ligand to neurotransmitters and induce hyperactivation of receptor-mediated signaling, leading to cell death. Chemical structures of metabolites were drawn with ChemDraw software.

potentially toxic metabolites in the cell are maintained at nontoxic levels either via further metabolism by metabolic enzymes or efficient secretion mechanisms. Demonstrating this notion, many inborn metabolic disorders such as phenylketonuria or maple syrup urine disease, which involve loss of function mutations in amino acid breakdown genes, are thought to be caused by toxic accumulation of amino acid degradation pathway metabolites, as dietary amino acid restriction can ameliorate or prevent the pathology [21, 22]. Here, we will discuss metabolites that are normally produced in our bodies and are thus endogenous, that also have documented toxic properties. We define "endogenous metabolites" as those that are annotated by Kyoto Encyclopedia of Genes and Genomes [23] as substrates or products of the 1900 metabolic enzymes encoded in our genome [24]. It is clear in the body of literature that there are documented toxic properties for many of these metabolites. Those defined toxic metabolites could be categorized based on how they confer the toxicity to the cells (Fig. 2); (1) ROS-producing metabolites, (2) reactive metabolites, (3) metabolite analogs, (4) excitotoxins, and (5) not established/unknown biology.

\section{ROS-producing metabolites}

The major intracellular sources of reactive oxygen species (ROS; $\mathrm{OH} \bullet, \mathrm{O}_{2}^{-\bullet}, \mathrm{H}_{2} \mathrm{O}_{2}$, etc.) are thought to be mitochondrial respiration and the activities of NADPH oxidase, 2oxoacid dehydrogenases, and superoxide dismutases 
(SOD1/2/3) [25]. In a normal state, the intracellular level of ROS is controlled in balance with intracellular antioxidants to protect cells from oxidative damage [25, 26]. It appears that intermediates of metabolic pathways can also directly or indirectly result in ROS production, which may account for their toxicity (Fig. 2a). For example, hydrogen selenide is an intermediate in the selenocysteine biosynthesis pathway, a pathway that incorporates the trace nutrient selenium to provide selenocysteine residues in 25 different selenoproteins in the human body. Inhalation of hydrogen selenide is known to be toxic [27], and it has been proposed that selenide reacts with water during its decay into elemental selenium, forming hydroxy radicals, superoxide and hydrogen peroxide [28], suggesting a potential mechanism for its toxicity. Bilirubin is a metabolite in the heme breakdown pathway, which occurs in the liver and is proposed to be the toxic component of jaundice [29]. While bilirubin can function as an antioxidant at high doses, it has also been shown to result in mitochondrial ROS accumulation [30,31], which promotes neuronal and cancer cell death $[32,33]$. Treatment of a high level of hypoxanthine, a product of the purine catabolism pathway, is also cytotoxic by creating superoxide radicals as it breaks down to xanthine and uric acid by xanthine oxidase [34, 35]. Polyamines in the arginine catabolic pathway such as agmatine [36, 37], spermidine [38], and spermine [39, 40] can directly bind to acidic sites in proteins and nucleic acids and also generate hydrogen peroxide when they are processed via a number of oxidases [41], which may account for their toxicity. 3-hydroxyanthranilate, an intermediate in the tryptophan degradation pathway, is known to kill $\mathrm{T}$ cells through ROS generation [42]. Autooxidation of the 3hydroxyanthranilate requires molecular oxygen, which produces superoxide radicals and hydrogen [43]. In addition, cysteamine, an amino thiol compound and an intermediate of the taurine synthesis pathway, has been shown to inhibit cell proliferation and survival and cancer cell invasion and metastasis at high levels [44, 45]. A proposed mechanism is that its thiol group can produce hydrogen peroxide upon reaction with transition metals [46].

\section{Reactive metabolites}

Some metabolites contain functional groups that are chemically reactive. As such, they can form adducts or react with macromolecules such as DNA and proteins, which can result in their detrimental modification and cellular toxicity (Fig. 2a). The aldehydes are molecules having at least one hydrogen atom substituent on the carbonyl carbon atom. These metabolites are toxic due to their electrophilicity by creating adducts with biological nucleophiles [47]. For example, formaldehyde, formed during the oxidative breakdown of the folate backbone, has the well-known toxic property of being able to efficiently crosslink biological macromolecules [20]. In addition, malondialdehyde and 4-hydroxynonenal (4-HNE), also known as lipid peroxidation markers because they are final products of polyunsaturated fatty acid peroxidation [48], can confer toxicity to cells by interacting with DNA and proteins [49-51]. Other lipid peroxidation products that have aldehyde groups, such as crotonaldehyde, 4oxononenal (4-ONE), and acrolein have been reported as toxic molecules [51-53]. Both 4-HNE and 4-ONE are potent cross-linkers of tubulin assembly, which results in impairment of tubulin function [51]. Acrolein forms DNA and protein adducts and promotes mitochondrial disruption, ER stress, and membrane damage at the cellular level [53]. The dopamine catabolic pathway metabolite 3,4dihydroxyphenylacetaldehyde is also a toxic aldehyde, as it modifies tyrosine hydroxylase and inhibits its activity [54]. As another example of aldehydes, methylglyoxal can be produced nonenzymatically as a byproduct of excessive glycolytic intermediates or glycine, and via other mechanisms, and is a potent toxic compound [55-58]. Propensity for methylglyoxal production may be high in cancer cells as its upstream sources of glycolysis and glycine production are upregulated in cancers. Showing another important aspect of methylglyoxal in tumor biology, regulatory myeloid cells suppress T-cell effector function via cell-cell transfer of methylglyoxal, aiding the immune evasion of tumors [59]. Beyond cancer biology, methylglyoxal is implicated as a pathological agent in diabetes and neurodegenerative disorders such as Alzheimer's and Parkinson's disease [58].

Several metabolites are toxic due to their strong tendency to react with free thiol groups of proteins or glutathione (Fig. 2a). For example, methacrylyl-CoA, an intermediate of the valine catabolic pathway, is proposed to be a toxic component of beta-hydroxyisobutyryl coenzyme A deacylase deficiency [60]. Methacrylyl-CoA readily reacts with free sulfhydryl groups, which results in cellular damage $[60,61]$. Glutaconyl-CoA, another intermediate of the valine catabolic pathway, has been suggested to be the main toxic component of the inborn disorder glutaryl-CoA dehydrogenase deficiency [62]. The addition product of glutaconyl-CoA to cysteine has been found in urine [62]. As another example of a reactive metabolite, xanthurenic acid, an end product of the tryptophan degradation pathway, is also toxic by covalently binding to proteins, which leads to protein unfolding, ER stress [63], and apoptosis [64].

Overall, many examples exist in the literature of metabolites that show reactivity toward biological macromolecules and thus exert their toxicity. In addition to further exploring the examples mentioned above, it may be possible in future efforts to predict toxic metabolites based on the 
presence of reactive groups, such as reactive aldehydes, and to explore these as a therapeutic strategy.

\section{Toxic analogs}

Many enzymes have the capacity to catalyze not just one standard reaction, but also physiologically irrelevant secondary reactions, referred to as "promiscuous" reactions $[65,66]$. These promiscuous reactions of enzymes have provided an opportunity to develop "antimetabolite" chemotherapeutic drugs such as nucleoside analogs [67] and glucose analogs [68]. These analogs compete with physiological glucose and nucleosides and bind to intracellular enzymes to induce cytotoxicity via behaving like antimetabolites. Interestingly, some findings support that the accumulation of endogenous metabolites can induce cell death by working as antimetabolites. For example, elevated dUTP/dTTP ratios can cause uracil misincorporation by DNA polymerase during DNA synthesis [69, 70] (Fig. 2b). The misincorporation of dUTP leads to DNA excision and strand breakage, which eventually leads to cell death [71]. Another example of a potential toxic metabolite analog is Sadenosylhomocysteine (SAH). SAH is a product of a methyltransferase reaction that uses $\mathrm{S}$-adenosylmethionine (SAM) as a substrate, and it is structurally analogous to SAM. It is well established that the ratio of SAM/SAH is an indicator of cellular methylation potential and SAH accumulation can inhibit the activity of many methyltransferases via feedback inhibition [72-75]. Supporting that the disrupted methylation status could be detrimental to cells, the accumulation of SAH was shown to be toxic to lymphoma and lymphoblastic cells [73, 74]. 2-Hydroxyglutarate (2$\mathrm{HG}$ ) is another example of a toxic analog metabolite, as it is chemically similar and acts as an analog to alphaketoglutarate $(\alpha-\mathrm{KG})$. 2-HG exists in D and L enantiomeric forms. D-2-HG is produced by neomorphic mutations in isocitrate dehydrogenase (IDH) 1 or 2 which causes them to aberrantly produce D-2-HG from $\alpha$-KG. D-2-HG in turn is an oncometabolite that is responsible for the oncogenic effects of IDH mutations. L-2-HG is formed via promiscuous, NADH-dependent substrate usage of lactate dehydrogenase $\mathrm{A}$ and malate dehydrogenase $1 / 2$, due to increased NADH/NAD + ratio, which occurs in hypoxia or electron transport chain dysfunction [76-78]. L-2-HG can also accumulate by defects in the L-2-HG processing enzyme L-2-hydroxyglutarate dehydrogenase (L2HGDH) and is the cause of the disorder 2-hydroxyglutaric aciduria [79]. Both of these forms can potentially be both oncogenic as well as toxic due to the inhibition of $\alpha$-KG-dependent histone and DNA demethylases and resulting changes in gene expression [80]. Indeed, the accumulation of L-2-HG, due to L2HGDH mutations or mitochondrial stress, exerts neuronal or glial dysfunction and toxicity in the central nervous system [81-83]. The prevention of L-2-HG overproduction through electron transport chain activity is also important for the normal function of hematopoietic stem cells and regulatory $\mathrm{T}$ cells $[77,78]$. It is intriguing to consider that some toxic metabolites that are endogenously produced in cancer cells may be used to poison them via a mechanism similar to the aforementioned examples of "antimetabolite" chemotherapeutics.

\section{Excitotoxins}

Neurotransmitters are endogenous chemicals that transmit signals from one neuron to another neuron, gland cell, or muscle cell [84]. The identified neurotransmitters are mostly amino acids, peptides, and monoamines, and their binding to the receptor can activate calcium influx and/or downstream signaling. Some endogenous metabolites have the potential to target neurotransmitter receptors, and they can excessively activate this process to toxic levels. Some toxic metabolites appear to exert toxicity by aberrantly triggering neurotransmitter receptors. For example, quinolinate, a downstream product of the kynurenine processing pathway, is a neuroactive metabolite. Quinolinic acid induces excitotoxic cell damage as an agonist for the N-methyl-Daspartate (NMDA) receptor (Fig. 2c), and additional mechanisms such as oxidative stress, cytoskeletal disruption, energetic dysfunction may also be involved [85, 86]. Glutaryl-CoA dehydrogenase deficiency, an inherited neurometabolic disorder, is characterized by the accumulation of glutaric acid and 3-hydroxyglutaric acid [87, 88]. The 3hydroxyglutaric acid induces cell death via NMDA receptor activation, and both glutaric acid and 3-hydroxyglutaric acid regulate glutamatergic and GABAergic neurotransmission, which leads to disrupted balance of excitatory and inhibitory neurotransmission [88]. As another example of a possible excitotoxin, homocysteine activates NMDA receptor-mediated signaling [89, 90]. Homocysteine also acts as a reactive metabolite by incorporating into protein via disulfide or homocysteinylation on amide groups, which affects protein structure and function [91]. While these toxic metabolites may exert toxicity in a neuronal context, some studies suggest that excitotoxic mechanisms can also affect cancer cells. It has been recognized that many types of cancer cells ectopically express neurotransmitters [92], and some studies indicate toxic effects of neurotransmitters/ excitotoxic metabolites such as dopamine and the aforementioned 3-hydroxyglutaric acid [93]. Undesirable toxicity to neurons is an obvious concern for strategies which aim to poison cancer cells with these types of toxic metabolites. It may nonetheless be feasible to explore such a therapeutic strategy for inducing accumulation of these metabolites in 
tumors outside the CNS, in which the drug cannot cross the blood brain barrier, thus avoiding CNS toxicity.

\section{Not established (unknown biology)}

Some metabolites have been known to have antiproliferative or toxic effects in cells, but their toxic mechanisms are not clearly understood. For example, dehydroepiandrosterone, an intermediate for steroid hormone synthesis, exerts growth inhibitory functions in hepatocytes [94], neuronal cells [95, 96], and breast and pancreatic cancer cells [97, 98]. The growth inhibitory effect of the dehydroepiandrosterone appears to involve perturbation of complex I of the mitochondrial respiratory chain [95], DNA synthesis [94], and SAM production and cardiolipin depletion [97], However, how these processes are inhibited are not clear. Ceramide, a central metabolite in sphingolipid metabolism, is well established as a tumor suppressor lipid and a cell death-inducing signal, and perhaps the best known "toxic metabolite" [99]. Ceramide has been shown to affect various processes including ERK and JNK signaling and modulation of apoptotic machinery [100], but the exact mechanism for how ceramide impacts these biological processes is still under debate. Accumulation of squalene, an intermediate in sterol biosynthesis, has been shown to be lipotoxic in yeast and cancer cells [101-103] by disturbing the maintenance of plasma membrane potential and increasing passive uptake [103], but further molecular characterizations are required. As another example, 4-methylthio-2-oxobutanoate, an intermediate in the methionine salvage pathway, has been shown to effectively induce apoptosis, and the underlying mechanism for this effect is unclear [104, 105]. Fumarylacetoacetate, a metabolite in the tyrosine degradation pathway that is thought to be the pathogenic metabolite in hereditary tyrosinemia [106, 107], has been shown to be both mutagenic and cytotoxic, eliciting deregulation of various biological processes such as glutathione depletion, ERK activation, and cytochrome c release [108, 109]. While some mechanisms such as reactivity with thiols and mitotic spindle disruption have been suggested [109], how fumarylacetoacetate interacts with these biological processes is not established. Another set of metabolites for which the mechanism of toxicity is incompletely understood are the gangliosides, sialic acid containing glycosphingolipids, which are documented to be toxic and are associated with inherited diseases due to its toxicity [110, 111]. While aberrant lysosomal accumulation and dysfunction, signaling dysfunction, and mechanisms related to lipid raft-like microdomains have been uncovered, the exact molecular nature of ganglioside toxicity remains to be found. While we are showing some selected examples, it appears that there are many reports of toxic effects of metabolites spanning various metabolic pathways, for which the reason for toxicity is undetermined. The fact that these metabolites can be toxic when at aberrantly high levels may indicate that they normally have previously unrecognized functions as regulatory components of biological processes or similarly interact with proteins or other biomolecules. An intriguing possibility is that, such as in ceramide's ability to act as a potent death signal, there may exist important signaling functions for many metabolites that are yet to be uncovered.

As seen across the body of literature and summarized above, a variety of metabolites can be toxic to cells through various molecular mechanisms known and unknown. There are likely many other metabolites and mechanisms of toxicity yet to be uncovered. These examples lead us to posit that many metabolic enzymes in cells may be important not necessarily for what they produce, but for what they get rid of.

\section{The kitchen sink and achieving cancer-specific toxicity}

As outlined in the previous section, many metabolites that are normally produced in cells have toxic properties, suggesting that their accumulation would be deleterious to a cell. This has an important implication for the function of metabolic enzymes - that enzymes that process a toxic metabolite in a pathway may serve an important detoxification function, as they prevent accumulation of toxic metabolites. This immediately suggests that it may be possible to induce poisoning of cancer cells with an accumulation of toxic metabolites that they themselves have produced, by targeting such "detoxification enzymes". Thus, the detoxifying enzymes represent novel and druggable targets for killing cancer cells.

But how can a therapeutic window exist for such a mechanism, so that the toxicity occurs primarily in cancer cells? In theory, the detoxification requirement would be in proportion to the production of the toxic metabolite in the first place. This can be analogized as a "kitchen sink" model (Fig. 3), where the faucet represents the upstream metabolic enzymes or transporters that are responsible for formation or import of the toxic metabolite, and the drain represents the "detoxifying" enzyme preventing the overflow of the sink with the toxic metabolite. In this model, the drain is only required in cells in which the faucet is on, i.e., the toxic metabolite is appreciably being produced. Thus, if certain cancer cells produce a particular toxic metabolite at a greater rate than normal cells (Fig. 3b, c), they should be more dependent on the detoxifying enzyme than normal cells, and drugs that target this detoxifying enzyme should be selectively toxic toward these cells.

Our recent findings on the selenocysteine biosynthesis pathway [112] illustrate and provide a proof of principle of 


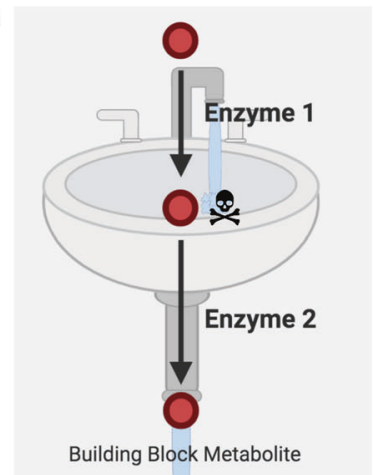

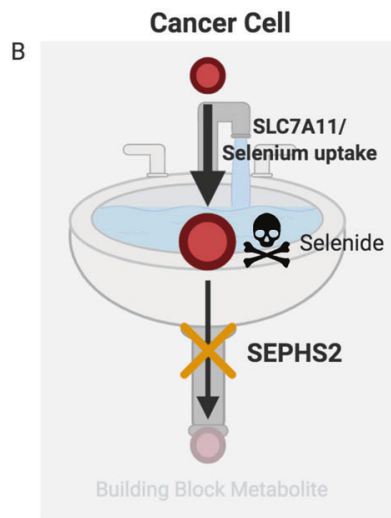

B

Fig. 3 Kitchen sink model for metabolic pathway containing toxic metabolite. Red dots and arrows represent metabolites and enzymatic reactions, respectively. Faucet and drain of kitchen sink are analogies of enzymatic reactions of upstream and downstream metabolic enzymes, respectively; the basin represents the accumulation level of the toxic metabolite. a The level of toxic metabolite candidate is constantly maintained at a nontoxic level as both faucet and drain are opened (active), balancing metabolite production and removal. b Example of toxic metabolite accumulation by targeting the downstream detoxification enzyme. SLC7A11 and SEPHS2 are the faucet and drain for toxic hydrogen selenide in the selenocysteine biosynthesis pathway. Many cancer cells have elevated SLC7A11 expression relative to normal cells and are able to import selenium and produce

the kitchen sink concept of selective toxicity. This metabolic pathway synthesizes selenocysteinyl-tRNA used in the production of 25 different selenoproteins encoded in the human genome, which contain one or more selenocysteine residues. A metabolic intermediate in this pathway, hydrogen selenide, is reported to be toxic $[27,113]$ and in the class of ROSproducing toxic metabolites (Table 1). We found that the sole enzyme that processes selenide-SEPHS2-is essential in a subset of cancer cell lines, including breast cancer and glioblastoma lines, but not in any nontransformed or primary lines tested. Investigating further, we were able to conclude that SEPHS2 is essential in these cell lines due to their elevated expression of SLC7A11, which induces the import of the dietary selenium compound selenite and its conversion to toxic selenide (Fig. 3b). Normal cells are spared, as they do not appreciably express SLC7A11, and thus do not depend on SEPHS2 detoxification (Fig. 3c). Importantly, we verified a toxic gain-of-function mechanism of toxicity based on selenide toxicity, as preemptive KO of SLC7A11, while also abrogating selenoprotein production similarly to SEPHS2 $\mathrm{KO}$, prevented the toxicity of SEPHS2 KO in cancer cells. This exemplifies a critical and robust test for identification of a toxic metabolite poisoning event: disruption of the upstream toxic metabolite producing step should rescue against the effects of detoxifying enzyme $\mathrm{KO}$, if the detoxifying enzyme was required for detoxification rather than what it produces (Fig. 3d).

Thus, the SLC7A11/selenide production/SEPHS2 detoxification axis illustrates a kitchen sink mechanism of
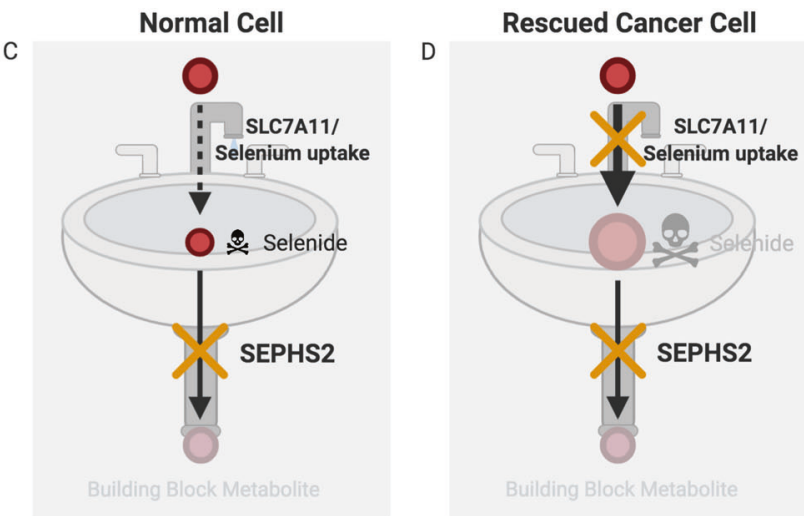

selenide at an elevated rate. Thus, their faucet is wide open, and disrupting SEPHS2 is akin to blocking the drain, resulting in toxic overflow. c However, in normal cells, disrupting SEPHS2/blocking the drain is not a problem as the faucet is relatively "closed". In both $\mathbf{b}$ and c, building block metabolite cannot be produced, yet only $\mathbf{b}$ is toxic, indicating that the toxicity must have come from toxic metabolite accumulation rather than consequences of downstream building block production. d Similarly, in a cancer cell such as in scenario b, the preemptive $\mathrm{KO}$ of the upstream transporter SLC7A11 rescues the cells against the toxic effects of SEPHS2 $\mathrm{KO}$, further demonstrating that toxicity in $\mathbf{b}$ was due to toxic metabolite accumulation. All diagrams were created with BioRender.com.

toxicity, and at the same time suggests potential advantages of a metabolite poisoning strategy to kill cancer cells. First, this pathway illustrates how in some instances the downstream building block product of a metabolic pathway per se may not be critical for the survival and proliferation of a cell, in contrast to the acute requirement for detoxification. Indeed, we demonstrated that the loss of all selenoproteins can be tolerated in normal cells indefinitely, whereas the toxic accumulation of selenide can acutely induce cell death in cancer cells. Second, it demonstrates how the factor that determines the requirement for detoxification is likely to be logical and predictable-in this case, the transporter involved in both selenium import and selenide production. This is a significant advantage, as one can easily predict which types of cancers may be amenable to SEPHS2 inhibitor treatment, either at the level of the cancer subtype or even at the individual patient level. This will help predict exactly which patients will benefit most from pharmacological inhibition of the detoxifying enzyme. Third, the level of toxic metabolite accumulation may be modulated by dietary input into the pathway, which can help increase or decrease efficacy to "dial in" the correct dosage of the toxic metabolite. We showed that supplementation with selenite in the tissue culture media synergistically enhanced the toxicity of SEPHS2 KO in cancer cells, suggesting that with this type of approach, pharmacological inhibition of the detoxifying enzyme may be combined with dietary supplementation with metabolic precursors to the toxic metabolite. Conversely, if the toxic 
Table 1 List of toxic metabolites.

\begin{tabular}{|c|c|c|c|}
\hline Category & Toxic metabolite & Related pathway & $\begin{array}{l}\text { Ref supporting toxic } \\
\text { property }\end{array}$ \\
\hline \multirow[t]{8}{*}{ ROSgens } & Hydrogen selenide & Selenocysteine synthesis & {$[27,28,113]$} \\
\hline & Bilirubin & Heme degradation & [29-33] \\
\hline & Hypoxanthine & Purine metabolism & {$[34,35]$} \\
\hline & Agmatine & Arginine catabolism & {$[36,37]$} \\
\hline & Spermidine & Arginine catabolism & {$[38,41]$} \\
\hline & Spermine & Arginine catabolism & {$[39-41]$} \\
\hline & 3-Hydroxyanthranilate & Tryptophan degradation & {$[42,43]$} \\
\hline & Cysteamine & Taurine synthesis & {$[44-46]$} \\
\hline \multirow{11}{*}{$\begin{array}{l}\text { Reactive metabolites } \\
\text { (Aldehydes, reactive to } \\
\text { thiols, etc.) }\end{array}$} & Formaldehyde & $\begin{array}{l}\text { By product of oxidative } \\
\text { demethylation/deamination }\end{array}$ & {$[20,115]$} \\
\hline & Malondialdehyde & Lipid peroxidation byproduct & [49] \\
\hline & 4-hydroxynonenal & Lipid peroxidation byproduct & {$[50,51]$} \\
\hline & Crotonaldehyde & Lipid peroxidation byproduct & {$[52]$} \\
\hline & 4-oxononenal & Lipid peroxidation byproduct & {$[51]$} \\
\hline & Acrolein & Lipid peroxidation byproduct & {$[53]$} \\
\hline & 3,4-dihydroxyphenylacetaldehyde & Dopamine degradation & {$[54]$} \\
\hline & Methylglyoxal & Various & [55-59] \\
\hline & Methacrylyl-CoA & Valine catabolism & {$[60,61]$} \\
\hline & Glutaconyl-CoA & Valine catabolism & {$[62]$} \\
\hline & Xanthurenic Acid & Tryptophan degradation & {$[63,64]$} \\
\hline \multirow[t]{3}{*}{ Toxic analogs } & dUTP & Nucleotide synthesis & {$[69-71]$} \\
\hline & S-adenosylhomocysteine & Transmethylation & {$[72-74]$} \\
\hline & L-2-hydroxyglutarate & $\begin{array}{l}\text { Promiscuous reaction of } \mathrm{LDH} \\
\text { and } \mathrm{MDH}\end{array}$ & [77-83] \\
\hline \multirow[t]{4}{*}{ Excitotoxins } & Quinolinic acid & Kynurenine degradation & {$[85,86]$} \\
\hline & 3-hydroxyglutaric acid & Kynurenine degradation & {$[87,88]$} \\
\hline & Glutaric acid & Kynurenine degradation & [88] \\
\hline & Homocysteine & Homocysteine synthesis & {$[89-91]$} \\
\hline \multirow[t]{8}{*}{ Not Established } & Dehydroepiandrosterone & Steroid hormone synthesis & [94-98] \\
\hline & Ceramide & Sphingolipid synthesis & {$[99,100]$} \\
\hline & Squalene & Steroid hormone synthesis & {$[101-103,116]$} \\
\hline & 4-methylthio-2-oxobutanoate & Methionine salvage pathway & {$[104,105]$} \\
\hline & Fumarylacetoacetate & Tyrosine degradation & [107-109] \\
\hline & Gangliosides & Ganglioside synthesis & {$[110,111]$} \\
\hline & ex) Ganglioside GM3 & Ganglioside synthesis & [117-119] \\
\hline & ex) Ganglioside GD3 & Ganglioside synthesis & {$[120-122]$} \\
\hline
\end{tabular}

metabolite accumulation from cancer cells is too great as to pose hazards to the patient, the toxicity may be tempered through dietary restriction of the upstream precursors. In conclusion, the toxic metabolite accumulation approach provides a method to induce acute poisoning to kill cancer cells, with additional potential advantages of predictability of susceptible cells and the opportunity for dietary modulation of the treatment.

\section{Discussions: caveats and a future blueprint for expansion}

We have discussed in "The kitchen sink and achieving cancerspecific toxicity" the concept, proof of principle, and advantages of a toxic metabolite accumulation strategy to kill cancer cells. As a novel concept, this approach holds promise for uncovering novel classes of cancer targets and even clinical implications for diseases beyond cancer; however, there are also important caveats to consider. Here, we will discuss how this approach can be expanded in a systematic manner, but with caution.

As described in "Toxic metabolites", examples of toxic metabolites can be found sprinkled throughout the literature across various fields. In each case, the downstream enzyme (s) that are responsible for processing these metabolites are "detoxifiers" and potential targets for inducing toxicity in cells, and this may be selective toward certain cancer cells if they have an increased production rate for the toxic metabolite. Thus, it will be useful to catalog an extensive list of toxic metabolites (the "endotoxome") and the set of downstream enzymes that process them. In this manner, once an extensive list has been curated, it may be possible to systematically analyze the metabolic activities of different cancers and use this to predict which toxic metabolite pathways can be targeted to achieve selective toxicity for a particular cancer. This sort of analysis may even be carried 
out on a patient to patient or individual tumor to tumor level based on gene expression analyses or metabolite profiling analyses of tumor biopsies. We predict that in the coming years many more studies and strategies involving individual detoxification enzymes may be uncovered, and over time systematic approaches may be developed.

An important caveat to a systematic identification of toxic metabolites is that it is hard to determine an exact threshold to define a toxic metabolite vs a nontoxic metabolite. Even relatively nonreactive, nontoxic metabolites may become toxic at high enough concentrations. For systematic cataloging of toxic metabolites, empirical dose response testing of each "endogenous metabolite" in purified form across several types of cell lines would allow one to set a dose-defined threshold or hierarchy of toxic metabolites. However, procuring an extensive library of metabolites in purified form presents a significant challenge to this approach. As an alternative approach, toxic metabolites may be "functionally" defined and cataloged using the "kitchen sink" model. As described in "The kitchen sink and achieving cancer-specific toxicity", if $\mathrm{KO}$ of a metabolic enzyme is deleterious, but can be rescued by the concomitant $\mathrm{KO}$ of the upstream enzyme (or transporter), this would indicate that the accumulation of the metabolite between those two enzymes was the cause for the toxicity (Fig. 3d). While functionally determining these cases represents a challenge in terms of experimental scale, mining the essentiality of enzymes in large-scale essentiality data resources such as the Cancer Cell Line Encyclopedia [114] may be a useful starting point.

A second caveat to be considered in future studies is the transport of toxic metabolites into and out of cells. Certain toxic metabolites may be incredibly toxic inside cells but may not be easily identified, as media treatment of the metabolite may not readily enter cells to exert a toxic effect. The same metabolites that are not easily transported into and out of a cell may represent attractive targets for therapy as they are "trapped" within the cell, as opposed to another metabolite that may be readily exported from the cell before toxic accumulation is achieved.

The third caveat is possible host toxicity. Even though the accumulation of a toxic metabolite should occur in a cancer cell-specific manner as described, it is possible that the toxic metabolites that accumulate in the tumor may spread to the host tissues. This risk further emphasizes the need to test examples of this approach in vivo, for example in tumor xenograft models. Conversely, the "spread" of toxicity may actually be a significant advantage in therapy when considering that tumors are known to be metabolically heterogeneous; thus, if a majority of tumor cells are selectively vulnerable to toxic metabolite accumulation, this may result in toxicity of neighboring invulnerable tumor cells, overcoming a universal limitation of chemotherapy.
Despite these potential caveats, the notion of poisoning cancer cells with their own metabolites represents an unconventional, underexplored, and potentially highly efficacious method for killing cancer cells in a predictive and selective manner. We hope that this review serves as a blueprint for other groups to determine whether toxic metabolite exploitation opportunities exist in their metabolic pathway of interest. Furthermore, the relevance of toxic metabolites may extend beyond simply being an exploitable tool in cancer therapy. Since toxic metabolites are normally produced, their presence-however transient -may contribute to cellular wear and tear mechanisms. The formation of aldehydes and ROS-producing metabolites, for example, if reacting with DNA, may contribute to the daily burden of DNA damage in a cell. If they react with proteins, this can result in protein dysfunction and even misfolding events. DNA damage and protein misfolding are events thought to be universally present (and pathologically contributing) to aging and age-dependent disorders such as Alzheimer's disease. Any deregulation of transcription, transport, or other biological functions that result in an imbalance between toxic metabolite production and detoxification may therefore increase the level of cellular damage over time in an organism. Toxic metabolites may represent a large and underappreciated group of metabolites that, over time, can contribute to a variety of disorders; thus, the premises mentioned here may be applicable across a range of diseases. In closing, toxic metabolites may lie at the interface of metabolism and disease in many regards, and as outlined here, cancer is a prime avenue in which they may be harnessed for therapeutic benefit.

Acknowledgements We sincerely apologize for not being able to add numerous important studies in our review due to space limitations. We thank Christopher Cervantes and David Sabatini for helpful discussions. All authors are supported by the Suh Kyungbae Foundation (SUHF) Young Investigator Award.

\section{Compliance with ethical standards}

Conflict of interest The authors declare that they have no conflict of interest.

Publisher's note Springer Nature remains neutral with regard to jurisdictional claims in published maps and institutional affiliations.

Open Access This article is licensed under a Creative Commons Attribution 4.0 International License, which permits use, sharing, adaptation, distribution and reproduction in any medium or format, as long as you give appropriate credit to the original author(s) and the source, provide a link to the Creative Commons license, and indicate if changes were made. The images or other third party material in this article are included in the article's Creative Commons license, unless indicated otherwise in a credit line to the material. If material is not included in the article's Creative Commons license and your intended use is not permitted by statutory regulation or exceeds the permitted use, you will need to obtain permission directly from the copyright 
holder. To view a copy of this license, visit http://creativecommons. org/licenses/by/4.0/.

\section{References}

1. Warburg O. On the origin of cancer cells. Science. 1956;123:309-14.

2. Vander Heiden MG, Cantley LC, Thompson CB. Understanding the Warburg effect: the metabolic requirements of cell proliferation. Science. 2009;324:1029-33.

3. Yang L, Venneti S, Nagrath D. Glutaminolysis: a hallmark of cancer metabolism. Annu Rev Biomed Eng. 2017;19:163-94.

4. Swinnen JV, Brusselmans K, Verhoeven G. Increased lipogenesis in cancer cells: new players, novel targets. Curr Opin Clin Nutr Metab Care. 2006;9:358-65.

5. Currie E, Schulze A, Zechner R, Walther TC, Farese RV Jr. Cellular fatty acid metabolism and cancer. Cell Metab. 2013; 18:153-61.

6. Fauman EB, Rai BK, Huang ES. Structure-based druggability assessment-identifying suitable targets for small molecule therapeutics. Curr Opin Chem Biol. 2011;15:463-8.

7. Xu D, Jalal SI, Sledge GW, Meroueh SO. Small-molecule binding sites to explore protein-protein interactions in the cancer proteome. Mol Biosyst. 2016;12:3067-87.

8. DeBerardinis RJ, Chandel NS. Fundamentals of cancer metabolism. Sci Adv. 2016;2:e1600200.

9. Luengo A, Gui DY, Vander Heiden MG. Targeting metabolism for cancer therapy. Cell Chem Biol. 2017;24:1161-80.

10. Farber S, Diamond LK. Temporary remissions in acute leukemia in children produced by folic acid antagonist, 4-aminopteroylglutamic acid. N Engl J Med. 1948;238:787-93.

11. Robinson AD, Eich ML, Varambally S. Dysregulation of de novo nucleotide biosynthetic pathway enzymes in cancer and targeting opportunities. Cancer Lett. 2020;470:134-40.

12. Vander Heiden MG. Targeting cancer metabolism: a therapeutic window opens. Nat Rev Drug Disco. 2011;10:671-84.

13. Locasale JW, Grassian AR, Melman T, Lyssiotis CA, Mattaini KR, Bass AJ, et al. Phosphoglycerate dehydrogenase diverts glycolytic flux and contributes to oncogenesis. Nat Genet. 2011; 43:869-74.

14. Flavin R, Peluso S, Nguyen PL, Loda M. Fatty acid synthase as a potential therapeutic target in cancer. Future Oncol. 2010; 6:551-62.

15. Kitatani K, Idkowiak-Baldys J, Hannun YA. The sphingolipid salvage pathway in ceramide metabolism and signaling. Cell Signal. 2008;20:1010-8.

16. Commisso C, Davidson SM, Soydaner-Azeloglu RG, Parker SJ, Kamphorst JJ, Hackett S, et al. Macropinocytosis of protein is an amino acid supply route in Ras-transformed cells. Nature. 2013;497:633-7.

17. Cantor JR, Abu-Remaileh M, Kanarek N, Freinkman E, Gao X, Louissaint A Jr, et al. Physiologic medium rewires cellular metabolism and reveals uric acid as an endogenous inhibitor of UMP synthase. Cell. 2017;169:258-72 e217.

18. Wexler P. TOXNET: an evolving web resource for toxicology and environmental health information. Toxicology. 2001;157:3-10.

19. Kim S, Thiessen PA, Bolton EE, Chen J, Fu G, Gindulyte A, et al. PubChem substance and compound databases. Nucleic Acids Res. 2016;44:D1202-13.

20. Burgos-Barragan G, Wit N, Meiser J, Dingler FA, Pietzke M, Mulderrig L, et al. Mammals divert endogenous genotoxic formaldehyde into one-carbon metabolism. Nature. 2017;548:549-54.

21. Blau N, van Spronsen FJ, Levy HL. Phenylketonuria. Lancet. 2010;376:1417-27.
22. Blackburn PR, Gass JM, Vairo FPE, Farnham KM, Atwal HK, Macklin S, et al. Maple syrup urine disease: mechanisms and management. Appl Clin Genet. 2017;10:57-66.

23. Kanehisa M, Goto S. KEGG: kyoto encyclopedia of genes and genomes. Nucleic Acids Res. 2000;28:27-30.

24. Possemato R, Marks KM, Shaul YD, Pacold ME, Kim D, Birsoy K, et al. Functional genomics reveal that the serine synthesis pathway is essential in breast cancer. Nature. 2011;476:346-50.

25. Sies H, Berndt C, Jones DP. Oxidative stress. Annu Rev Biochem. 2017;86:715-48.

26. Sarniak A, Lipinska J, Tytman K, Lipinska S. Endogenous mechanisms of reactive oxygen species (ROS) generation. Postepy Hig Med Dosw. 2016;70:1150-65.

27. Schecter A, Shanske W, Stenzler A, Quintilian H, Steinberg H. Acute hydrogen selenide inhalation. Chest. 1980;77:554-5.

28. Peyroche G, Saveanu C, Dauplais M, Lazard M, Beuneu F, Decourty L, et al. Sodium selenide toxicity is mediated by O2dependent DNA breaks. PLoS ONE. 2012;7:e36343.

29. Dean E. Neonatal jaundice. Nurs Stand. 2016;30:15.

30. NaveenKumar SK, Thushara RM, Sundaram MS, Hemshekhar M, Paul M, Thirunavukkarasu C, et al. Unconjugated Bilirubin exerts pro-apoptotic effect on platelets via p38-MAPK activation. Sci Rep. 2015;5:15045.

31. Fujiwara R, Haag M, Schaeffeler E, Nies AT, Zanger UM, Schwab M. Systemic regulation of bilirubin homeostasis: potential benefits of hyperbilirubinemia. Hepatology. 2018;67:1609-19.

32. Cesaratto L, Calligaris SD, Vascotto C, Deganuto M, Bellarosa $\mathrm{C}$, Quadrifoglio F, et al. Bilirubin-induced cell toxicity involves PTEN activation through an APE1/Ref-1-dependent pathway. J Mol Med. 2007;85:1099-112.

33. Rawat V, Bortolussi G, Gazzin S, Tiribelli C, Muro AF. Bilirubin-induced oxidative stress leads to DNA damage in the cerebellum of hyperbilirubinemic neonatal mice and activates DNA double-strand break repair pathways in human cells. Oxid Med Cell Longev. 2018;2018:1801243.

34. Iwata K, Shibuya H, Ohkawa Y, Inui N. Chromosomal aberrations in V79 cells induced by superoxide radical generated by the hypoxanthine-xanthine oxidase system. Toxicol Lett. 1984; 22:75-81.

35. Ryu HM, Kim YJ, Oh EJ, Oh SH, Choi JY, Cho JH, et al. Hypoxanthine induces cholesterol accumulation and incites atherosclerosis in apolipoprotein E-deficient mice and cells. J Cell Mol Med. 2016;20:2160-72.

36. Wolf C, Bruss M, Hanisch B, Gothert M, von Kugelgen I, Molderings GJ. Molecular basis for the antiproliferative effect of agmatine in tumor cells of colonic, hepatic, and neuronal origin. Mol Pharm. 2007;71:276-83.

37. Mayeur C, Veuillet G, Michaud M, Raul F, Blottiere HM, Blachier F. Effects of agmatine accumulation in human colon carcinoma cells on polyamine metabolism, DNA synthesis and the cell cycle. Biochim Biophys Acta. 2005;1745:111-23.

38. Dewey DL. Methyl glyoxal bis guanylhydrazone abolition of the toxic action of spermidine on cells in culture. Cancer Lett. 1979;6:247-50.

39. Brunton VG, Grant MH, Wallace HM. Spermine toxicity and glutathione depletion in BHK-21/C13 cells. Biochem Pharm. 1990;40:1893-900.

40. Huber M, Poulin R. Antiproliferative effect of spermine depletion by $\mathrm{N}$-cyclohexyl-1,3-diaminopropane in human breast cancer cells. Cancer Res. 1995;55:934-43.

41. Pegg AE. Toxicity of polyamines and their metabolic products. Chem Res Toxicol. 2013;26:1782-800.

42. Hiramatsu R, Hara T, Akimoto H, Takikawa O, Kawabe T, Isobe $\mathrm{K}$, et al. Cinnabarinic acid generated from 3-hydroxyanthranilic acid strongly induces apoptosis in thymocytes through the 
generation of reactive oxygen species and the induction of caspase. J Cell Biochem. 2008;103:42-53.

43. Dykens JA, Sullivan SG, Stern A. Oxidative reactivity of the tryptophan metabolites 3-hydroxyanthranilate, cinnabarinate, quinolinate and picolinate. Biochem Pharm. 1987;36:211-7.

44. Fujisawa T, Rubin B, Suzuki A, Patel PS, Gahl WA, Joshi BH, et al. Cysteamine suppresses invasion, metastasis and prolongs survival by inhibiting matrix metalloproteinases in a mouse model of human pancreatic cancer. PLoS ONE. 2012;7:e34437.

45. Jeitner TM, Renton FJ. Inhibition of the proliferation of human neural neoplastic cell lines by cysteamine. Cancer Lett. 1996;103:85-90.

46. Jeitner TM, Lawrence DA. Mechanisms for the cytotoxicity of cysteamine. Toxicol Sci. 2001;63:57-64.

47. LoPachin RM, Gavin T. Molecular mechanisms of aldehyde toxicity: a chemical perspective. Chem Res Toxicol. 2014;27:1081-91.

48. Esterbauer H, Cheeseman KH. Determination of aldehydic lipid peroxidation products: malonaldehyde and 4-hydroxynonenal. Methods Enzymol. 1990;186:407-21.

49. Del Rio D, Stewart AJ, Pellegrini N. A review of recent studies on malondialdehyde as toxic molecule and biological marker of oxidative stress. Nutr Metab Cardiovasc Dis. 2005;15:316-28.

50. Shoeb M, Ansari NH, Srivastava SK, Ramana KV. 4Hydroxynonenal in the pathogenesis and progression of human diseases. Curr Med Chem. 2014;21:230-7.

51. Stewart BJ, Doorn JA, Petersen DR. Residue-specific adduction of tubulin by 4-hydroxynonenal and 4-oxononenal causes crosslinking and inhibits polymerization. Chem Res Toxicol. 2007; 20:1111-9.

52. Lee SE, Park YS. Role of lipid peroxidation-derived alpha, betaunsaturated aldehydes in vascular dysfunction. Oxid Med Cell Longev. 2013;2013:629028.

53. Moghe A, Ghare S, Lamoreau B, Mohammad M, Barve S, McClain C, et al. Molecular mechanisms of acrolein toxicity: relevance to human disease. Toxicol Sci. 2015;143:242-55.

54. Mexas LM, Florang VR, Doorn JA. Inhibition and covalent modification of tyrosine hydroxylase by 3,4-dihydroxyphenylacetaldehyde, a toxic dopamine metabolite. Neurotoxicology. 2011;32:471-7.

55. Kim D, Fiske BP, Birsoy K, Freinkman E, Kami K, Possemato $\mathrm{RL}$, et al. SHMT2 drives glioma cell survival in ischaemia but imposes a dependence on glycine clearance. Nature. 2015; 520:363-7.

56. Luengo A, Abbott KL, Davidson SM, Hosios AM, Faubert B, Chan $\mathrm{SH}$, et al. Reactive metabolite production is a targetable liability of glycolytic metabolism in lung cancer. Nat Commun. 2019;10:5604.

57. Kalapos MP. Methylglyoxal toxicity in mammals. Toxicol Lett. 1994;73:3-24.

58. Schalkwijk CG, Stehouwer CDA. Methylglyoxal, a highly reactive dicarbonyl compound, in diabetes, its vascular complications, and other age-related diseases. Physiol Rev. 2020;100:407-61.

59. Baumann T, Dunkel A, Schmid C, Schmitt S, Hiltensperger M, Lohr K, et al. Regulatory myeloid cells paralyze T cells through cell-cell transfer of the metabolite methylglyoxal. Nat Immunol. 2020;21:555-66.

60. Brown GK, Hunt SM, Scholem R, Fowler K, Grimes A, Mercer $\mathrm{JF}$, et al. beta-hydroxyisobutyryl coenzyme A deacylase deficiency: a defect in valine metabolism associated with physical malformations. Pediatrics. 1982;70:532-8.

61. Taniguchi K, Nonami T, Nakao A, Harada A, Kurokawa T, Sugiyama $\mathrm{S}$, et al. The valine catabolic pathway in human liver: effect of cirrhosis on enzyme activities. Hepatology. 1996; 24:1395-8.
62. Lehnert W, Sass JO. Glutaconyl-CoA is the main toxic agent in glutaryl-CoA dehydrogenase deficiency (glutaric aciduria type I). Med Hypotheses. 2005;65:330-3.

63. Malina HZ. Xanthurenic acid provokes formation of unfolded proteins in endoplasmic reticulum of the lens epithelial cells. Biochem Biophys Res Commun. 1999;265:600-5.

64. Malina HZ, Richter C, Mehl M, Hess OM. Pathological apoptosis by xanthurenic acid, a tryptophan metabolite: activation of cell caspases but not cytoskeleton breakdown. BMC Physiol. 2001;1:7.

65. Copley SD. Shining a light on enzyme promiscuity. Curr Opin Struct Biol. 2017;47:167-75.

66. Jeffryes J, Strutz J, Henry C, Tyo KEJ. Metabolic in silico network expansions to predict and exploit enzyme promiscuity. Methods Mol Biol. 2019;1927:11-21.

67. Galmarini CM, Mackey JR, Dumontet C. Nucleoside analogues and nucleobases in cancer treatment. Lancet Oncol. 2002; 3:415-24.

68. Zhang D, Li J, Wang F, Hu J, Wang S, Sun Y. 2-Deoxy-Dglucose targeting of glucose metabolism in cancer cells as a potential therapy. Cancer Lett. 2014;355:176-83.

69. Ingraham HA, Tseng BY, Goulian M. Nucleotide levels and incorporation of 5-fluorouracil and uracil into DNA of cells treated with 5-fluorodeoxyuridine. Mol Pharm. 1982;21:211-6.

70. Kennedy EM, Daddacha W, Slater R, Gavegnano C, Fromentin $\mathrm{E}$, Schinazi RF, et al. Abundant non-canonical dUTP found in primary human macrophages drives its frequent incorporation by HIV-1 reverse transcriptase. J Biol Chem. 2011;286:25047-55.

71. Curtin NJ, Harris AL, Aherne GW. Mechanism of cell death following thymidylate synthase inhibition: 2'-deoxyuridine-5'triphosphate accumulation, DNA damage, and growth inhibition following exposure to CB3717 and dipyridamole. Cancer Res. 1991;51:2346-52.

72. Richon VM, Johnston D, Sneeringer CJ, Jin L, Majer CR, Elliston $\mathrm{K}$, et al. Chemogenetic analysis of human protein methyltransferases. Chem Biol Drug Des. 2011;78:199-210.

73. Kredich NM, Hershfield MS. S-adenosylhomocysteine toxicity in normal and adenosine kinase-deficient lymphoblasts of human origin. Proc Natl Acad Sci USA. 1979;76:2450-4.

74. Kredich NM, Martin DV Jr. Role of S-adenosylhomocysteine in adenosinemediated toxicity in cultured mouse $\mathrm{T}$ lymphoma cells. Cell. 1977;12:931-8.

75. Kennedy BP, Bottiglieri T, Arning E, Ziegler MG, Hansen LA, Masliah E. Elevated S-adenosylhomocysteine in Alzheimer brain: influence on methyltransferases and cognitive function. J Neural Transm (Vienna). 2004;111:547-67.

76. Intlekofer AM, Dematteo RG, Venneti S, Finley LW, Lu C, Judkins AR, et al. Hypoxia induces production of L-2Hydroxyglutarate. Cell Metab. 2015;22:304-11.

77. Weinberg SE, Singer BD, Steinert EM, Martinez CA, Mehta MM, Martinez-Reyes I, et al. Mitochondrial complex III is essential for suppressive function of regulatory $\mathrm{T}$ cells. Nature. 2019;565:495-9.

78. Anso E, Weinberg SE, Diebold LP, Thompson BJ, Malinge S, Schumacker PT, et al. The mitochondrial respiratory chain is essential for haematopoietic stem cell function. Nat Cell Biol. $2017 ; 19: 614-25$.

79. Kranendijk M, Struys EA, Salomons GS, Van der Knaap MS, Jakobs C. Progress in understanding 2-hydroxyglutaric acidurias. J Inherit Metab Dis. 2012;35:571-87.

80. Ye D, Guan KL, Xiong Y. Metabolism, activity, and targeting of D- and L-2-hydroxyglutarates. Trends Cancer. 2018;4:151-65.

81. Rzem R, Achouri Y, Marbaix E, Schakman O, Wiame E, Marie $\mathrm{S}$, et al. A mouse model of L-2-hydroxyglutaric aciduria, a disorder of metabolite repair. PLoS ONE. 2015;10:e119540. 
82. Rzem R, Veiga-da-Cunha M, Noel G, Goffette S, Nassogne MC, Tabarki B, et al. A gene encoding a putative FAD-dependent L-2hydroxyglutarate dehydrogenase is mutated in L-2-hydroxyglutaric aciduria. Proc Natl Acad Sci USA. 2004;101:16849-54.

83. Hunt RJ, Granat L, McElroy GS, Ranganathan R, Chandel NS, Bateman JM. Mitochondrial stress causes neuronal dysfunction via an ATF4-dependent increase in L-2-hydroxyglutarate. J Cell Biol. 2019;218:4007-16.

84. Hyman SE. Neurotransmitters. Curr Biol. 2005;15:R154-8.

85. Guillemin GJ. Quinolinic acid, the inescapable neurotoxin. FEBS J. 2012;279:1356-65.

86. Braidy N, Grant R, Adams S, Brew BJ, Guillemin GJ. Mechanism for quinolinic acid cytotoxicity in human astrocytes and neurons. Neurotox Res. 2009;16:77-86.

87. Latini A, Borba Rosa R, Scussiato K, Llesuy S, Bello-Klein A, Wajner M. 3-Hydroxyglutaric acid induces oxidative stress and decreases the antioxidant defenses in cerebral cortex of young rats. Brain Res. 2002;956:367-73.

88. Kolker S, Koeller DM, Okun JG, Hoffmann GF. Pathomechanisms of neurodegeneration in glutaryl-CoA dehydrogenase deficiency. Ann Neurol. 2004;55:7-12.

89. Choudhury S, Borah A. Activation of NMDA receptor by elevated homocysteine in chronic liver disease contributes to encephalopathy. Med Hypotheses. 2015;85:64-7.

90. Bleich S, Degner D, Sperling W, Bonsch D, Thurauf N, Kornhuber J. Homocysteine as a neurotoxin in chronic alcoholism. Prog Neuropsychopharmacol Biol Psychiatry. 2004;28:453-64.

91. Perla-Kajan J, Twardowski T, Jakubowski H. Mechanisms of homocysteine toxicity in humans. Amino Acids. 2007;32:561-72.

92. Jiang SH, Hu LP, Wang X, Li J, Zhang ZG. Neurotransmitters: emerging targets in cancer. Oncogene. 2020;39:503-15.

93. Sarkar C, Chakroborty D, Chowdhury UR, Dasgupta PS, Basu S. Dopamine increases the efficacy of anticancer drugs in breast and colon cancer preclinical models. Clin Cancer Res. 2008;14:2502-10.

94. Rao MS, Subbarao V. Dehydroepiandrosterone inhibits DNA synthesis of rat hepatocytes induced by partial hepatectomy or mitogen (ciprofibrate). Cell Prolif. 1997;30:1-5.

95. Safiulina D, Peet N, Seppet E, Zharkovsky A, Kaasik A. Dehydroepiandrosterone inhibits complex I of the mitochondrial respiratory chain and is neurotoxic in vitro and in vivo at high concentrations. Toxicol Sci. 2006;93:348-56.

96. Gil-ad I, Shtaif B, Eshet R, Maayan R, Rehavi M, Weizman A. Effect of dehydroepiandrosterone and its sulfate metabolite on neuronal cell viability in culture. Isr Med Assoc J. 2001;3:639-43.

97. Cheng ML, Shiao MS, Chiu DT, Weng SF, Tang HY, Ho HY. Biochemical disorders associated with antiproliferative effect of dehydroepiandrosterone in hepatoma cells as revealed by LCbased metabolomics. Biochem Pharm. 2011;82:1549-61.

98. Lopez-Marure R, Contreras PG, Dillon JS. Effects of dehydroepiandrosterone on proliferation, migration, and death of breast cancer cells. Eur J Pharm. 2011;660:268-74.

99. Ogretmen B, Hannun YA. Biologically active sphingolipids in cancer pathogenesis and treatment. Nat Rev Cancer. 2004; 4:604-16.

100. Ueda N. Ceramide-induced apoptosis in renal tubular cells: a role of mitochondria and sphingosine-1-phoshate. Int $\mathrm{J}$ Mol Sci. 2015;16:5076-124.

101. Mahoney CE, Pirman D, Chubukov V, Sleger T, Hayes S, Fan $\mathrm{ZP}$, et al. A chemical biology screen identifies a vulnerability of neuroendocrine cancer cells to SQLE inhibition. Nat Commun. 2019;10:96.

102. Valachovic M, Garaiova M, Holic R, Hapala I. Squalene is lipotoxic to yeast cells defective in lipid droplet biogenesis. Biochem Biophys Res Commun. 2016;469:1123-8.

103. Csaky Z, Garaiova M, Kodedova M, Valachovic M, Sychrova H, Hapala I. Squalene lipotoxicity in a lipid droplet-less yeast mutant is linked to plasma membrane dysfunction. Yeast. 2020; $37: 45-62$.

104. Tang B, Kadariya Y, Murphy ME, Kruger WD. The methionine salvage pathway compound 4-methylthio-2-oxobutanate causes apoptosis independent of down-regulation of ornithine decarboxylase. Biochem Pharm. 2006;72:806-15.

105. Quash G, Roch AM, Chantepie J, Michal Y, Fournet G, Dumontet C. Methional derived from 4-methylthio-2-oxobutanoate is a cellular mediator of apoptosis in BAF3 lymphoid cells. Biochem J. 1995;305(Pt 3): 1017-25.

106. Russo P, O'Regan S. Visceral pathology of hereditary tyrosinemia type I. Am J Hum Genet. 1990;47:317-24.

107. Li L, Zhang Q, Yang H, Zou Q, Lai C, Jiang F, et al. Fumarylacetoacetate hydrolase knock-out rabbit model for hereditary tyrosinemia type 1. J Biol Chem. 2017;292:4755-63.

108. Kubo S, Sun M, Miyahara M, Umeyama K, Urakami K, Yamamoto T, et al. Hepatocyte injury in tyrosinemia type 1 is induced by fumarylacetoacetate and is inhibited by caspase inhibitors. Proc Natl Acad Sci USA. 1998;95:9552-7.

109. Jorquera R, Tanguay RM. Fumarylacetoacetate, the metabolite accumulating in hereditary tyrosinemia, activates the ERK pathway and induces mitotic abnormalities and genomic instability. Hum Mol Genet. 2001;10:1741-52.

110. Breiden B, Sandhoff K. Ganglioside metabolism and its inherited diseases. Methods Mol Biol. 2018;1804:97-141.

111. Regier DS, Proia RL, D'Azzo A, Tifft CJ. The GM1 and GM2 gangliosidoses: natural history and progress toward therapy. Pediatr Endocrinol Rev. 2016;13(Suppl 1):663-73.

112. Carlisle AE, Lee N, Matthew-Onabanjo AN, Spears ME, Park $\mathrm{SJ}$, Youkana D, et al. Selenium detoxification is required for cancer-cell survival. Nat Metab. 2020;2:603-11.

113. Banerjee BD, Dwivedi S, Singh S. Acute hydrogen selenide gas poisoning admissions in one of the hospitals in Delhi, India: case report. Hum Exp Toxicol. 1997;16:276-8.

114. Ghandi M, Huang FW, Jane-Valbuena J, Kryukov GV, Lo CC, McDonald ER 3rd, et al. Next-generation characterization of the Cancer Cell Line Encyclopedia. Nature. 2019;569:503-8.

115. Tulpule K, Dringen R. Formaldehyde in brain: an overlooked player in neurodegeneration? J Neurochem. 2013;127:7-21.

116. Brown AJ, Chua NK, Yan N. The shape of human squalene epoxidase expands the arsenal against cancer. Nat Commun. 2019;10:888.

117. Zhao HY, Ma YP. [Apoptosis effects of GM3 ganglioside on U266 cells and its possible mechanism]. Zhongguo Shi Yan Xue Ye Xue Za Zhi. 2018;26:1022-6.

118. Choi HJ, Chung TW, Kang SK, Lee YC, Ko JH, Kim JG, et al. Ganglioside GM3 modulates tumor suppressor PTEN-mediated cell cycle progression-transcriptional induction of p21(WAF1) and p27(kip1) by inhibition of PI-3K/AKT pathway. Glycobiology. 2006;16:573-83.

119. Yoshinaga A, Kajiya N, Oishi K, Kamada Y, Ikeda A, Chigwechokha PK, et al. NEU3 inhibitory effect of naringin suppresses cancer cell growth by attenuation of EGFR signaling through GM3 ganglioside accumulation. Eur $\mathrm{J}$ Pharm. 2016;782:21-9.

120. Kang NY, Kang SK, Lee YC, Choi HJ, Lee YS, Cho SY, et al. Transcriptional regulation of the human GD3 synthase gene expression in Fas-induced Jurkat T cells: a critical role of transcription factor NF-kappaB in regulated expression. Glycobiology. 2006;16:375-89.

121. Sorice M, Garofalo T, Misasi R, Manganelli V, Vona R, Malorni W. Ganglioside GD3 as a raft component in cell death regulation. Anticancer Agents Med Chem. 2012;12:376-82.

122. Malisan F, Testi R. GD3 ganglioside and apoptosis. Biochim Biophys Acta. 2002;1585:179-87. 